\title{
Germanica
}

\section{Le poète prend le train.}

Paysages, rêveries et mythologies ferroviaires dans la poésie suédoise

Wenn der Dichter in den Zug steigt. Landschaft, Träumerei und

Eisenbahnmythologie in der schwedischen Dichtung

\section{Sylvain Briens}

\section{(2) OpenEdition}

12 Journals

Édition électronique

URL : http://journals.openedition.org/germanica/2185

DOI : 10.4000/germanica.2185

ISSN : 2107-0784

Éditeur

Université de Lille

\section{Édition imprimée}

Date de publication : 31 décembre 2001

Pagination : $37-53$

ISBN : 9782913857063

ISSN : 0984-2632

Référence électronique

Sylvain Briens, «Le poète prend le train. », Germanica [En ligne], 29 | 2001, mis en ligne le 20 septembre 2013, consulté le 06 octobre 2020. URL : http://journals.openedition.org/germanica/2185 ; DOI :

https://doi.org/10.4000/germanica.2185

Ce document a été généré automatiquement le 6 octobre 2020.

(c) Tous droits réservés 


\section{Le poète prend le train.}

Paysages, rêveries et mythologies ferroviaires dans la poésie suédoise

Wenn der Dichter in den Zug steigt. Landschaft, Träumerei und

Eisenbahnmythologie in der schwedischen Dichtung

\section{Sylvain Briens}

Järnvägar och aldrig annat än järnvägar ! Den dagen kommer även för oss, ni kan tryggt lita därpå, och den är kanske närmare än man tror.

Vi skola icke mer äga någon av våra gamla hederliga landsvägar i behåll, ångan skall icke längre nöja sig med att plaska på våra sjöar, den skall börja spatsera omkring även på landbacken, land och rike skall beläggas med patenterade järnskenor, hela Europa, hela världen skola täckas med ett nät av järnvägar, en spindelväv, som industrien spinner kring vår arma planet, för att hålla bättre fast sitt rov, sedan hon väl fått det i sina klor ${ }^{1}$.

(Le chemin de fer, et jamais rien d'autre que le chemin de fer!

Ce jour arrivera même pour nous. Vous pouvez en être sûrs, et plutôt qu'on ne le pense. Nous n'allons plus conserver aucune de nos bonnes vieilles routes, la vapeur ne va plus se contenter de barboter sur les lacs, mais va commencer à se

promener sur la terre ferme, le pays va être équipé de rails brevetés, toute l'Europe, le monde entier, vont être couverts d'un réseau de chemins de fer, une toile d'araignée que l'industrie tisse 
autour de notre pauvre planète afin de mieux tenir sa proie, une fois prise dans ses griffes.)

1 Par ces quelques lignes, O.P. Sturzen-Becker (1811-1969) évoque un bouleversement majeur dans la culture suédoise, l'avènement du chemin de fer. Voici une invention qui transforme le paysage, bouscule les mentalités et métamorphose le rapport de l'homme au monde. Une véritable culture du voyage naît, la culture du nomadisme ferroviaire. Et la poésie suédoise, faisant pénétrer le lecteur dans les tourbillons d'une société bouleversée par l'innovation technique, se fait le porte-parole fidèle de cette modernité. Quelle est alors la place du train dans la création poétique suédoise?

\section{Un nouveau paysage vu de la fenêtre du train}

2 Le train prend réellement son essor en Suède, comme moyen de transport public, à la fin du dix-neuvième siècle. La poésie suédoise de cette époque, fascinée par le romantisme allemand et toute entière déterminée par la culture suédoise, place la nature au centre de son inspiration. Elle se peuple de paysages et le chemin de fer y entre par un biais tout à fait particulier, par une perspective dont l'enjeu poétique est immense : il transforme le regard du poète sur la nature.

\section{À la découverte de la Suède et de sa nature}

3 Dans un pays à l'étendue géographique considérable - la Suède s'étire sur mille cinq cent soixante quinze kilomètres du Nord au Sud - et aux contraintes climatiques difficiles, le développement du chemin de fer est fondamental pour les transports. Pour la première fois, les poètes découvrent les territoires du Nord de la Suède, jusque-là peu explorés. L'époque est au nationalisme et les poètes suédois saisissent l'occasion pour s'exalter de la richesse esthétique de leur pays. Les poèmes du train s'inscrivent dans ce mouvement de reconquête de l'identité nationale, quête qui passe par la réappropriation du territoire et de sa diversité géographique. Le train est une formidable occasion de se réconcilier avec les sources de la culture suédoise et de se glorifier de l'immense beauté de sa nature. Enthousiasmés par leurs découvertes ferroviaires, les écrivains ont alors décrit, dans ses moindres détails, la diversité et la richesse de la nature suédoise. Les forêts et les lacs de chaque région sont immortalisés par leurs plumes et les lecteurs les ont découverts par les livres sans même avoir à se déplacer. Remarquons que l'office national du tourisme suédois lance dans les années quarante une campagne « Känn ditt land» (Découvre ton pays), suite logique d'un élan déjà promu par les écrivains.

Ainsi les poètes partent-ils en train à la recherche d'une Suède, non pas symbolique mais bien réelle, cette Suède dont ils sont si fiers. Vingt ans après l'ouverture de la première ligne de chemin de fer en Suède ${ }^{2}$, August Strindberg (1849-1912) consacre déjà sa plume à la description des paysages suédois vus du train. Il s'agit de la première partie du long poème Nuits de somnambule par jours éveillés ${ }^{3}$. Le poète quitte la Suède. La première nuit du récit rapporte son voyage en train de Stockholm au sud de la Scanie. Alors que le train passe par la plaine scanienne, le narrateur remonte par la pensée le long de la voie de chemin de fer qu'il a parcourue.

I stjärnklar natt under halvtänd måne

han ser det sköna, det rika Skåne 


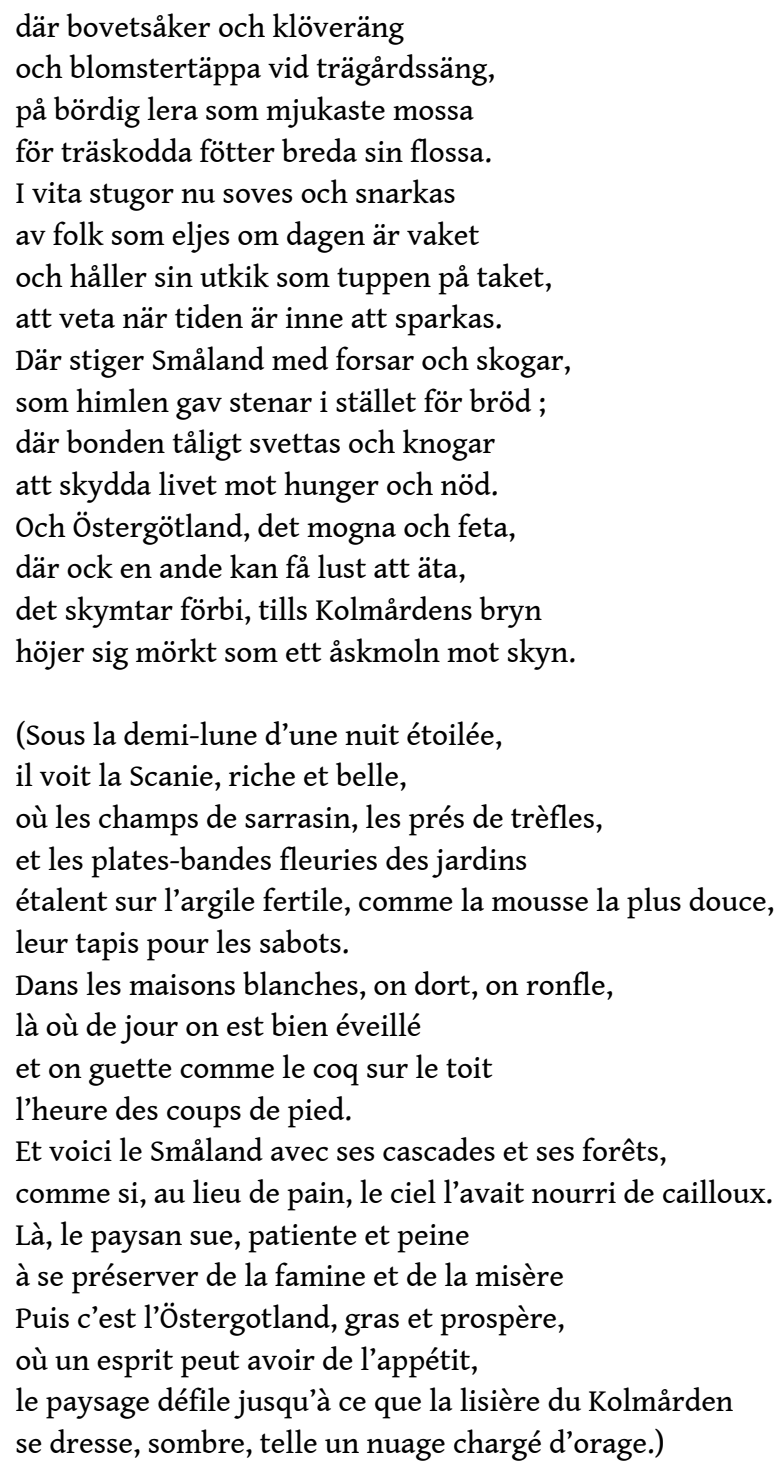

5 August Strindberg peint ici avec enthousiasme les régions traversées par le train. Chaque région, la Scanie, le Småland ou l'Östergötland, est décrite selon ses caractéristiques, ses richesses et ses particularités. Et chaque tableau panoramique est une invitation au voyage grâce à laquelle le lecteur part à la découverte de la Suède $\mathrm{e}^{4}$. Le poème Sur les rails ${ }^{5}$ de Bo Setterlind (1923-), de par sa description d'une action unique "Tu prends le train » - est un autre exemple de l'attachement de la poésie du train à décrire les espaces ruraux suédois. Voyages à travers la Suède, ces poèmes sont autant de témoignages géographiques à valeur documentaire. La poésie ferroviaire peint les espaces de la Suède rurale qui défilent à la fenêtre du train.

Prenons par exemple un aspect caractéristique de la nature suédoise, la dépendance générique du paysage aux différentes saisons. Si les poètes cherchent à décrire la Suède et ses paysages variés, ils doivent prendre en compte le cycle des saisons. C'est ainsi que des poèmes comme Voyageur ${ }^{6}$ de Birger Norman (1914-1995), Dans le train StockholmBoden $^{7}$ d'Artur Landfors, Train de jour ${ }^{8}$ de Lars Lundkvist (1906-1991), Réflexion ${ }^{9}$ de Lennart Frick (1939-), Le train de nuit du Nord ${ }^{10}$ de Harry Blomberg (1893-1950), La gare (11 $^{11}$ de Karl Asplund (1890-), ou encore Tour d'un soir ${ }^{12}$ de Sture Axelson (1913-) se fixent sur la thématique des voyages rythmés par les saisons. Le voyage en train y est donc 
l'occasion d'observer des paysages où les saisons posent leurs griffes. Les deux pôles hiver/été sont exploités dans leur sens large, tout d'abord l'hiver, avec sa nuit qui peut être longue d'une demi-année, d'une demi-vie, accompagnée de la neige dont la richesse évocatrice est immense, puis le contraste violent de l'été, qui n'est que renaissance, lumière et ivresse.

Le modèle pictural semble donc s'imposer dans la poésie du train. Les voyages rapides à travers la Suède inspirent à l'homme moderne un sentiment particulier par rapport à son pays, un pays réel et non plus symbolique qui s'étire devant ses yeux au rythme des saisons. On assiste à un mouvement littéraire qui s'attache à découvrir la richesse des paysages suédois et c'est la Suède rurale remplie de contrastes de lumière et d'obscurité qui s'en trouve soulignée. On voit que le train participe tout entier à une poétique de la Suède et de sa nature.

\section{L'écran du cinéma ferroviaire : un panorama à grande vitesse}

Certes, le train permet aux écrivains de voyager à travers la Suède et donc de la découvrir, mais il introduit également un extraordinaire changement de référentiel : en montant dans le train, l'observateur abandonne un regard statique sur la nature pour s'émerveiller devant un paysage défilant tout à coup sous ses yeux. L'influence de cette mobilité sur le paysage romantique de la nature suédoise est considérable, dans la mesure où elle crée de nouveaux paysages et transforme les paysages déjà connus. La fenêtre du wagon, telle le cadre d'un tableau, devient en quelques années, le point de vue préféré des poètes. Le déplacement du train donne vie et mouvement à leurs descriptions. Il n'est donc plus question de rêverie statique, ni de symbolisme abstrait. L'époque est à la mobilité et le poète doit se faire nomade. Le train se transforme en salle de cinéma ambulante, phénomène que souligne Verner von Heidenstam ${ }^{13}$ :

Vi tro ej längre poeten som rundar

en sirlig dikt på sitt rum en kväll,

när han kallar dessa stenrös fjäll

och fänadshagarna lundar.

(Nous ne croyons plus au poète qui peaufine un poème gracieux dans sa chambre un soir, quand il appelle ces cairns montagnes et ces petits enclos bosquets.)

Il se crée une nouvelle réciprocité, de sujet à objet, entre l'auteur et le paysage extérieur. Et les images idylliques de la nature, tant recherchées par les romantiques, doivent laisser place aux images mobiles. Même si les poètes ne sont évidemment pas en permanence assis dans le train, la vitesse les a transformés et leur poésie s'est mise en mouvement. C'est bien une rupture littéraire avec la poésie de la fin du XIX siècle. En train, le poète explore à présent l'espace à grande vitesse et cette circulation entraîne une délocalisation de l'intérêt du poète : délaissant les descriptions détaillées, il tourne sa plume vers les vues panoramiques qui défilent devant ses yeux.

Étudions les diverses facettes de cette mise en mouvement, véritable dynamisation poétique de la nature. Tout d'abord, les poètes prennent la mesure de l'intérêt stylistique du mouvement pour donner une image vivante de la nature dans leurs poèmes. Le train rythme en effet la narration et son déplacement guide les yeux du poète le long de la voie. De ce fait, il introduit dans la description un élément 
dynamique, comme un fil directeur qui nourrit la poétique. Le deuxième enjeu du mouvement est purement visuel. Il s'agit d'utiliser les effets de la vitesse. Deux exemples illustrent parfaitement comment la poésie a su exploiter la vitesse dans les descriptions de paysage, la première nuit de Nuits de somnambule par jours éveillés ${ }^{14}$ d'August Strindberg et Le courrier Stenbock ${ }^{15}$ de Carl Snoilsky (1814-1903). Passionnés par les temps modernes, ces deux écrivains remarquent, lors de voyages en train, comment les arbres apparaissent comme des éclairs à la fenêtre du wagon. Il n'est pas étonnant de retrouver le poème d'August Strindberg. La vitesse y est soulignée par la condensation géographique. Comme nous l'avons vu précédemment, les différentes régions entre Stockholm et la Scanie sont décrites dans une temporalité réduite et apparaissent en l'espace de quelques vers. La technique se fonde sur une sorte d'impressionnisme brut, enregistrant simultanéité et coïncidence. Il s'agit d'un poème ferroviaire, où le train devient - comme la caméra du cinéaste - le point mobile qui entraîne dans son rythme haletant un flot d'images. Le poète-voyageur semble pénétrer la signification des choses et accéder à la profondeur du réel. La technique utilisée par August Strindberg tient bien davantage de l'instantanéité que de celle du peintre. Une obsession de la vitesse, du mouvement, de ce que l'on aperçoit furtivement, imprègne cette poésie.

11 Ainsi les poètes du début du siècle montent dans le train et se réservent les places à la fenêtre. Ils s'abandonnent alors à la description de la nature scandinave et font du train le lieu où le paysage est roi. Le train devient un médiateur incontournable entre le poète et la nature. Du train, le poète jouit de la beauté de la nature et communie avec elle. Remarquons que, sur l'écran du cinéma ferroviaire, un thème domine largement, la nuit. Elle est présente dans de nombreux poèmes, inlassablement associée au train, sous des facettes contrastées, obscure ou claire, noire ou blanche. Malgré la rupture entre romantisme et réalisme, un thème cher aux romantiques a résisté aux évolutions : les nuits d'été et leur lumière, leitmotiv qui habite également la poésie du train. Les lacs et les forêts ont toujours fait partie de ces nuits blanches ; à présent on peut entendre au loin siffler un train, mais l'atmosphère reste identique, intérieure, mélancolique et peuplée de souvenirs.

\section{Train, rêverie et mélancolie}

12 Si les poètes sont partis en voyage, ils ont également invité le lecteur à faire partie de l'aventure. Le plus souvent dans la poésie du train, le lecteur ne regarde pas le poète voyager mais voyage avec lui. Nous avons étudié, dans le point précédent, la place du modèle pictural dans la poésie du train et la prédilection des poètes-voyageurs pour la description des paysages défilant à la fenêtre du wagon. De nombreux poètes mêlent à la description une analyse de l'activité perceptive du protagoniste devant les images qui défilent. Ils sont sensibles à la manière dont le voyageur découvre le paysage et rapportent ce qu'il en perçoit. Ceci crée un contraste par rapport à la primauté de la description dans les poèmes mentionnés précédemment. Ce n'est plus la spécificité des espaces qui est le centre de la poétique mais la structure même de l'expérience. Les écrivains soulignent la sensation du voyageur face à un monde éclaté et parcouru trop vite. Et pour la première fois dans la littérature suédoise, nous éprouvons le sentiment d'un mécanisme universel: la belle machinerie humaine (le train) conjuguée à la 
respiration d'un homme (le poète) qui nous confie, au rythme de ses trépidations, ses frémissements les plus secrets.

\section{L'atmosphère intériorisée du wagon}

13 Bercé pendant des heures par la cadence du train, le voyageur assis dans le train près de la fenêtre et contemplant le paysage se laisse bien évidemment surprendre par la rêverie. Dans les poèmes, la description du décor laisse alors place au voyage intérieur. Les déplacements effectifs se doublent de déplacements par la pensée qui font apparaître, dans l'espace réel du poème, un espace imaginaire qui s'emboîte dans le premier. Plusieurs éléments du train invitent à la méditation. Attardons-nous un instant sur ces catalyseurs de rêverie.

14 En premier lieu, le confort du wagon permet au voyageur de se détendre et l'incite à s'assoupir. Réflexion ${ }^{16}$ de Lennart Frick nous parle de cette libre réflexion du voyage, sans toutefois s'attarder sur son contenu. Le train y représente une sorte de sécurité, car il suit les rails, et le voyageur se laisse transporter avec passivité. Au confort du wagon s'ajoute le confort mental : l'itinéraire est sous la responsabilité du personnel de la compagnie de chemin de fer et l'heure d'arrivée est connue d'avance. La passivité du voyageur est donc totale et ce dernier peut laisser libre cours à ses pensées.

En outre, l'univers acoustique, propice en raison de sa régularité à la méditation, enveloppe le voyageur et l'isole du monde extérieur au train. La cadence donnée par le frottement régulier des roues sur les rails berce le voyageur-poète, et l'âme de ce dernier en profite pour s'échapper. Le poème Le train de nuit $d u$ Nord ${ }^{17}$ de Harry Blomberg ${ }^{18}$ est ponctué par le "dunkedunk dunkedi», bruit sourd du train en mouvement qui rythme la réflexion du voyageur.

Och jag lyss till tågets rytm och trampar takten med min sko

och jag drömmer och jag glömmer hela världen i min ro

och jag lutar mig tillbaka

emot bräderna som skaka

och jag sluter mina ögon i stilla blund och vaka.

Och till dikt och melodi blir det dova dunkedi

och det drömögt ljusa landet som vi rinna snabbt förbi

och det svarta tågets brus

och den natt som tänder ljus

kring de sovande människornas hus

(J'écoute le rythme du train et avec ma chaussure je bats la mesure,

je rêve et dans ma quiétude j'oublie le monde entier,

je m'adosse

aux planches de bois qui tremblent,

je ferme les yeux et m'assoupit tout en restant éveillé.

Le sourd dunkedi se fait musique et poésie,

ce pays clair comme un rêve que nous traversons rapidement,

le bruit du train noir,

la nuit qui éclaire

les maisons des gens endormis.)

Le voyageur s'assoupit inévitablement. Ses sens, ses souvenirs, sa volonté, ses pensées, et son âme enfin, ne lui laissent aucune alternative, l'heure est à la détente et à la rêverie. La résonance du «dunkedunk dunkedi » accompagne le voyageur, lui impose une cadence et s'immisce harmonieusement dans sa rêverie. «dunkedunk dunkedi» 
est un leitmotiv lancinant qui obsède le narrateur et rythme le poème, comme s'il devenait le battement du coeur du voyageur. Dans un autre poème, Le train de nuit ${ }^{19}$, Harry Blomberg exploite le lien entre décor sonore et rêverie du voyageur. Le «dunkedunk dunkedi» du poème précédent devient dans ce poème une mélodie se propageant dans la nature le long des voies ferrées. Et la symphonie ferroviaire inspire des sentiments méditatifs. Isolé des bruits du monde extérieur, à l'écoute du battement régulier du train sur les rails, le voyageur est habité par la sensation d'être dans un rêve, seul, loin de toute réalité.

\section{Un temps et un espace modifiés}

17 Lieu d'introspection, le train modifie le temps et l'espace. Lancé dans sa course, le train atteint un état permanent où le temps semble suspendu. À vitesse constante, il devient comme immobile, et, dans cette mobilité pure, l'espace se comprime tandis que le temps se détend. Le voyageur, seul face à lui-même dans une temporalité distendue et une spatialité condensée, fait l'expérience existentielle de la durée. Le voyage en train devient alors porteur d'imaginaire. August Strindberg nous offre, toujours dans le même poème, Nuits de somnambule par jours éveillés ${ }^{20}$, une métaphore explicite de l'influence de la transformation du temps et de l'espace sur la psychologie du voyageur. Alors que le corps, attaché au train, se déplace vers le sud, l'âme se détache du corps et s'envole vers le nord.

Men frigjord ande, den stackars trötte, som sina vingar så ofta nötte i flykt mot himlen, som redan befanns bestå av en färglös men vägbar substans av syre och kväve i behaglig blandning, ohimmelsk nog, men lämplig för andning, den stackars anden han glömmer programmet det nya-nya om materie och kraft, och blåser från fjädrarne järnvägsdammet ; som om aldrig annat än vingar han haft han kastar sig ut i den dunkla natten och svävar bort över land och vatten. Mot norr går färden luftig och kort, men kroppen skaks mot södern bort.

(Mais l'esprit libéré, fatigué le pauvre, qui tant de fois utilisa ses ailes, en fuite vers le ciel, un ciel qui se révéla déjà composé d'une substance incolore mais pondérable, mélange agréable d'azote et d'oxygène, fort peu céleste mais bon à respirer, le pauvre esprit, oublie le programme, le dernier cri en énergie et matière, et souffle la poussière du chemin de fer de ses plumes. Comme s'il n'eut jamais rien d'autre que ses ailes, il se jette dans la nuit noire et survole terres et eaux. C'est vers le nord que son voyage se dirige, léger et rapide, tandis que le corps est secoué vers le sud.)

18 Le corps reste dans le chaos oppressant du wagon, tandis que l'esprit s'envole avec légèreté, contemple le paysage et vole en contresens du train. Par cette image poétique 
de l'ascèse, l'auteur illustre parfaitement l'association intime du train et de la rêverie. L'âme se sépare du corps car le voyageur fait dans le train l'expérience d'un tempsespace différent. August Strindberg utilise la séparation ascétique du corps et de l'âme comme la figure allégorique de la dichotomie ferroviaire du temps-espace.

\section{Le contenu des rêveries ferroviaires : la nostalgie d'une enfance perdue}

Intéressons-nous maintenant au contenu de ces libres réflexions ferroviaires, plus proches de la rêverie irrationnelle que de la méditation contrôlée. Elles ont presque toutes en commun le thème de la vie qui passe et de la vie passée; au mouvement spatial du train répond le déplacement dans le temps du voyageur. Dans le poème Kalmar-Borås ${ }^{21}$, Lennart Sjögren (1930-) évoque l'ambiance méditative du voyage, où temps et espace se mélangent et où passé et futur s'intercalent. Le voyage en train est un moment de pause dans la temporalité où le voyageur prend une distance par rapport au réel. Cette distance lui donne l'occasion de se pencher sur sa propre vie comme si elle lui était devenue extérieure. La rupture spatio-temporelle du train engendre un dédoublement entre le voyageur assis dans le wagon et ce même voyageur dans la vie hors du train. De ce fait, la rêverie du poète-voyageur se peuple naturellement de souvenirs.

Aussi n'est-il pas surprenant que les poètes choisissent fréquemment de décrire les voyages qui les ramènent vers le pays de leur enfance, mise en scène qui ajoute une densité émotive à l'introspection du poète se penchant sur son passé. Dans son poème Dans le train Stockholm-Boden ${ }^{22}$, Artur Landfors nous conduit en Norrland, sa région natale. C'est une introspection où le poète se réconcilie avec ses origines géographiques, qui nous est proposée :

Vad detta vinterlandskap ändå drog,

dess smala vägar mellan gråa gårdar.

De vägarna var mina, det var där

så många kälkars spår jag också trampat.

Och varje rök, som från skorsten steg,

den hälsade mig nu : « här är du hemma ».

Vart fönster dunkla ruta sade mig :

« här är ditt land, din bygd, här hör du hemma ».

De många åren uti främmad land

nu som en drömbild plånades de ut.

De svåra ungdomsårens bitterhet,

dess armod, oro, längtan voro glömda.

Det var som om jag velat stanna där

och känna foten glida i ett spår,

som kälken gjort, där vägen gled förbi.

(Ce paysage traçait quand même

son chemin étroit à travers les fermes grises !

Ces chemins étaient les miens, c'était là

que j'avais marché le long de nombreuses traces de luge.

Et chaque nuage de fumée qui sortait de la cheminée

me saluait : « Te voici chez toi.»

Chaque fenêtre au carreau sombre me disait :

«Voici ton pays, ton village, ta place est ici. »

Les longues années passées dans les contrées lointaines, 
comme un rêve s'effacèrent.

L'amertume de ces années difficiles de jeunesse,

toute la misère, les soucis et la nostalgie étaient oubliés.

C'était comme si j'avais voulu rester là

et sentir mon pied glisser dans la trace

marquée par la luge, là où le chemin passait.)

Tous les éléments du paysage, le chemin tracé par le train, les nuages de fumée, les fenêtres, insistent sur l'appartenance du poète à ce pays, à ce village. Les racines du voyageur sont profondément ancrées dans cette région et effacent le temps de l'exil. Hemma (chez soi) se rapporte ici à la région de son enfance. En route vers sa région natale, le voyageur se retrouve face à son enfance, dans le pays des souvenirs. Placer le voyage en train sur un trajet connu confère au poème une densité géographique : à mesure que le train se rapproche de la destination, les paysages sont de plus en plus familiers, et l'émotion d'autant plus intense. Ainsi, dans le poème Le train de nuit du $N_{0} d^{23}$ de Harry Blomberg évoqué précédemment, hemma acquiert une valeur profonde. Il se transforme en refrain d'une chanson joyeuse qui libère le poète de la pesanteur de son présent.

Il est possible de trouver une explication à la fréquente association entre la rêverie du train et le pays natal. Le train a autrefois participé à la rupture géographique avec le pays d'origine. Si on y retourne en train, c'est en train qu'on l'avait auparavant quitté. Arrêtons-nous un instant sur la signification de l'enfance dans la culture suédoise, car cette dernière attache une forte importance à l'enfance perdue. Et l'enfance est intimement associée à un lieu particulier, hemma. La langue suédoise est riche en vocabulaire évoquant cet attachement. À ce lieu est en effet associé un endroit en particulier, regorgeant de souvenirs ensoleillés de l'enfance heureuse : smultronstället, le jardin secret, celui des fraises sauvages. Et puis, il y a la douloureuse perte de l'enfance, lorsque l'on est forcé de quitter la maison familiale. Un sentiment reflète cette rupture toute la vie, längtan, sorte de mélancolie d'un passé perdu ${ }^{24}$. Dans ce contexte affectif, le train s'intègre au cœur de la culture suédoise: il provoque la rupture géographique par rapport au lieu de l'enfance et cristallise de ce fait la perte de l'enfance.

La métaphore ferroviaire est explicite dans le poème Voyageur ${ }^{25}$ de Birger Norman. De même qu'il est impossible de retourner en enfance, le train n'atteindra jamais sa destination. Le narrateur exprime un goût désenchanté d'amertume et de tristesse mêlé au sentiment du jamais plus. Le lieu qu'il aimait n'est plus accessible, car il a changé, non pas de location géographique, mais d'ambiance. Le mot suédois stämning qui caractérise le rapport entre l'homme et un espace, y prend toute sa signification. Le lieu n'appartient à l'homme qu'à un moment donné. Quand le temps est passé, même si ce lieu signifie toujours beaucoup pour lui, il en est comme dépossédé. Ce symptôme est, selon le poète Birger Norman, caractéristique de tout voyageur de retour à son pays. Le train ressemble par cet aspect au corbeau d'Edgar Poe qui rappelle inlassablement au poète l'irréversibilité du temps qui passe.

De même Harry Blomberg, dans Train de nuit ${ }^{26}$, se bat avec ses souvenirs et ce terrible sentiment du temps qui passe inéluctablement. Assis confortablement dans le train, il se souvient de ses premiers voyages. C'était l'été, il était pauvre mais libre. À présent, c'est l'automne, et la richesse ne compense pas la jeunesse. Le train incite directement le poète à se remémorer le passé car chaque voyage en train est une occasion de comparer le voyage présent aux voyages effectués par le passé. Le train est comme une 
horloge et chaque voyage sonne implacablement l'heure, telle l'horloge de Baudelaire qui murmure "souviens-toi ». L'analogie entre les années passées et les trains empruntés appuie la métaphore. Ainsi, dans Arioso ${ }^{27}$ d'Erik Lindegren (1910-1968), une fois tous les trains partis, les horloges s'arrêtent. Toujours, on regrette le voyage précédent, alors que le printemps dispensait sa douceur, ou le voyage effectué voilà quelques années, lorsqu'on était jeune et bohème. Harry Blomberg utilise dans Train de $n u i t^{28}$ l'image d'un voyage à la destination inconnue pour exprimer ce sentiment de nostalgie que le narrateur, impuissant face à son destin, ne finit pas de ressentir.

De par son confort et son univers sonore ainsi que par la transgression du temps et de l'espace qu'il implique, le train est le lieu poétique de la rêverie par excellence, et la rêverie se peuple le plus souvent de souvenirs. L'âme s'évade lors des voyages en train. Le thème poétique le plus exploité reste la méditation sur l'enfance perdue, lors de voyages qui ramènent au pays natal. Parallèlement à ce thème, se développe une nouvelle dimension poétique autour du train, la mythologie ferroviaire, que nous nous proposons à présent d'étudier.

\section{Mythologies ferroviaires}

\section{La vitesse, déesse ou démon}

Le début du siècle, bouleversé par les innovations techniques, voit se développer une frénésie artistique autour des machines. L'esprit moderne est né. Les futuristes italiens, suivis par l'avant-garde parisienne, s'exaltent du dynamisme universel qui caractérise l'ère des machines. Avec la naissance d'un nouveau mythe, celui de la vitesse, les jeunes artistes de l'avant-garde suédoise, commencent eux aussi la recherche d'images capables de représenter ou d'exprimer le rythme vertigineux des temps modernes. La locomotive lancée à toute vitesse est le symbole tutélaire de la machine en mouvement et le poète suédois du début du siècle glorifie sa puissance mécanique.

Les changements du rapport au temps et à l'espace liés à l'avènement du train prennent principalement leur source dans la transformation de la notion de vitesse et les bouleversements littéraires qui leur sont associés s'attachent naturellement à décrire cette nouvelle déesse. Aussi effrayante qu'envoûtante, sa présence dans la poésie n'est pas neutre. Les poètes suédois ont témoigné de l'importance de ce nouvel être-dans-letemps et être-dans-l'espace en créant une mythologie de la vitesse. Il y a dans la poésie du train une notion de l'espace dévoré et de l'ivresse du glissement. Le voyageur est grisé par cette sensation, et fort du mouvement spatial et de la perte des repères qu'il implique, le voyageur subit un trouble mental, ce que Roland Barthes appelle, en parlant du pilote d'avion à réaction, « la crise immobile de la conscience corporelle ${ }^{29}$. Ainsi, Arnold Ljungdal (1901-1968), dans le poème Vitesse ${ }^{30}$, métamorphose le train en un monstre sacré au pouvoir incommensurable. Dans sa course effrénée, il emporte tout sur son passage, et, dévoreur avide, il avale puis rejette tous les éléments du paysage. C'est la vitesse, monstre implacable, qui prend le contrôle du train dont toute la mécanique semble s'emballer. La locomotive est une machine d'autant plus vivante qu'elle est rapide. De machine elle devient bête monstrueuse et incontrôlable, puis déesse noire, vampire avide de gravier ou démon tellurique. Le sublime naît de cette métamorphose de la machine, par définition non animée, en force naturelle voire même surnaturelle. C'est alors que se produit une osmose entre la machine et la nature. 
Dans le poème Succession au trône ${ }^{31}$, Gustav Fröding (1860-1911) développe une mythologie encore plus explicite. Autour du chemin de fer se construit une nouvelle cosmogonie : Chronos, monté sur son cheval, a été détrôné par son fils, un jeune roi se déplaçant en train. C'est la vitesse à présent qui gouverne le monde et le train est son serviteur.

Den gamle kung Kronos är död, hans son,

som ärvde hans tron, är ung,

och hastig till sinnes och lätt på tån,

är nittonde seklets kung.

Den gamle var känd som en patriark,

en stillsam och tyst nomad,

som strövade kring över livets mark,

beskedlig och jämn och glad.

Den unge är fallen för äventyr,

på vägar dem själv han byggt

vid svindlande bråddjup han trotsigt styr

sitt susande iltågs flykt.

(Le vieux roi Chronos est mort. C'est son fils

qui a hérité du trône.

Il est jeune, vif d'esprit et agile,

il est le roi du dix-neuvième siècle.

Le vieux roi était reconnu comme un patriarche,

un nomade calme et silencieux,

qui errait dans le domaine de la vie,

débonnaire, d'humeur égale, joyeux.

Le jeune roi est enclin à l'aventure,

sur les chemins qu'il a lui-même construits,

aux abrupts vertigineux, il conduit avec obstination

sa fuite frémissante de train express.)

\section{Le stade religieux}

Il faut souligner qu'avec l'avènement du train le sens profond de l'être-dans-le-temps et de l'être-dans-l'espace change, et l'espace poétique en est bouleversé. Le train est une cosmogonie refermée sur elle-même, avec une temporalité et une spatialité propre. Un poème d'Ola Hansson (1860-1925), Pensées d'un train de nuit ${ }^{32}$, illustre bien ce phénomène.

Det eviga glider, men stelnar ej -

panta rei.

Sekunden är livet, dygnet ej -

panta rei.

Ditt färgspel lever, din massa ej -

panta rei.

Du själv är Kosmos, materien ej -

panta rei.

(L'éternel glisse, mais ne se fige pas -

panta rei

La seconde est la vie, pas le jour - 
panta rei

Ton jeu de couleur vit, pas ta masse corporelle -

panta rei

Toi, tu es le Cosmos, pas la matière -

panta rei)

L'image d'Héraclite, panta rei (tout coule), rappelle que le temps passe et transforme tout. Le voyageur ressent pleinement ce temps qui passe à mesure que le train glisse. Dans la rêverie du voyageur, le temps devient le symbole de la vie. Le voyageur s'identifie à l'espace et se trouve face à quelque chose d'absolu. Certains poètes pensent à l'éternel, comme Ola Hansson; d'autres se tournent vers Dieu, comme August Strindberg dont le voyage des Nuits de somnambule par jours éveillés ${ }^{33}$ finit dans une église où l'esprit s'adresse à Dieu. Le voyage en train est vécu ici comme une démarche initiatique.

Pour comprendre cette réaction littéraire face au train, il faut avoir en mémoire le contexte intellectuel scandinave de la fin du dix-neuvième siècle, fortement imprégné de la philosophie de Kierkegaard. En effet, la transgression du temps à laquelle le train invite ressemble étrangement à l'image de la transcendance du temps que prône Kierkegaard. Le philosophe dégage trois stades sur le chemin de la vie, le stade esthétique, le stade éthique et le stade religieux. Le voyage en train peut être mis en parallèle avec ce troisième stade. L'échec de l'instant présent - qui caractérise le stade esthétique - est symbolisé par le départ, ou plus exactement par le désir de départ, tandis que l'échec de la projection - qui caractérise le stade éthique - réside dans le fait que le train n'a pas encore atteint sa destination, et ne l'atteindra pas réellement. Il reste donc au voyageur une seule solution, le voyage, ou plus exactement la pénétration dans le voyage en lui-même. Cette étape de méditation, nécessairement mystique selon Kierkegaard, correspond au stade religieux, réponse à l'échec des deux stades précédents. Aussi n'est-il pas étonnant de voir la rêverie d'August Strindberg arriver dans une église, face à Dieu. Pour de nombreux écrivains scandinaves, marqués par la philosophie de Kierkegaard, l'instant du voyage est profondément mystique.

\section{Conclusion}

Le train, dont la fenêtre offre au voyageur un panorama ambulant, métamorphose le spectacle de la nature en laissant le voyageur découvrir des régions jusque-là inconnues et en transformant son regard. À la contemplation immédiate du paysage s'ajoute l'absorption gourmande des éléments du paysage défilant à la vitesse du train. Le grondement inlassable des roues sur les rails rythme l'ennui confortable du voyage et incite à la rêverie nonchalante, une rêverie qui glisse le plus souvent vers la nostalgie du temps révolu de l'enfance. Enfin, bouleversés dans leur expérience du temps et de l'espace, les poètes s'exaltent des sensations de la vitesse, maintenant accessible à tous. De cette expérience, naît une mythologie du train, machine à la fois magique et mystérieuse. Marquant ainsi le triomphe de l'homme sur la nature, le train rapproche le poète de Dieu.

Par conséquent, avec l'avènement du chemin de fer, une transformation majeure s'opère: le train permet d'assigner à la configuration spatio-temporelle dans le discours littéraire une fonction centrale et décisive. Et sa présence dans la littérature est une véritable leçon d'initiation aux secrets du monde moderne. Soulignons pour 
finir que la poésie du train constitue une littérature à proprement parler, car à sa thématique spécifique s'ajoutent de nouveaux outils littéraires. Le train apporte à la poésie un nouveau chronotope, profondément moderne. De ce fait, il bouleverse les indices culturels de la vie et de la mort et, par un renversement de métaphore, en devient les métaphores. L'enjeu du train est alors dramatique : le train se fait théâtre d'actes humains, théâtre tout à la fois moderne et respectueux des règles de la tragédie classique, unité de lieu, de temps et d'action.

\section{NOTES}

1. Sturzen-Becker, O.P. : Med en bit blyerts, Stockholm : Bonnier, 1848, 121 p.

2. En 1862 s'ouvre la ligne Stockholm-Göteborg.

3. Strindberg, A. : Sömngångarnätter på vakna dagar, Stockholm : Bonnier, 1884, 94 p. (traduction française par Jean de Faramond, Paris : Librairie Séguin, 1990).

4. Selma Lagerlöf exploite le même principe dans Le merveilleux voyage de Nils Holgersson à travers la Suède, en utilisant le point de vue panoramique du dos de l'oie pour décrire chaque région de Suède.

5. Setterlind, B. : På rälsen, Det ringer på dörren, Stockholm : Bonnier, 1976, 101 p.

6. Norman, B. : Resenär, Våg under vinden, Stockholm : FIB : s lyrikklubb, 1957, 75 p.

7. Landfors, A. : Pa tåget Stockholm-Boden, Träd som bara grönska, Stockholm : FIB : s lyrikklubb, 1962, $93 \mathrm{p}$.

8. Lundkvist, L. : Dagtåg, Koöga, Stockholm : Norstedt, 1977, 130 p.

9. Frick, L. : Besinning, Vindsvalt, Stockholm : Geber, 1960, 28 p.

10. Blomberg, H. : Norrgående nattåget, Stora orons män, Stockholm : Bonnier, 1918, 114 p.

11. Asplund, K. : Stationen, Daphne, Stockholm : Norstedt, 1921, 101 p.

12. Axelson, S. : Aftontur, Lyktor om aftonen, Stockholm, 1945, $58 \mathrm{p}$.

13. Von Heidenstam, V. : Tiveden, Dikter, Stockholm : Bonnier, 1940, 426 p.

14. Op. cit.

15. Snoilsky, C. : Stenbocks kurir, Svenska bilder, Stockholm : Bonnier, 1894, 89 p.

16. Op. cit.

17. Op. cit.

18. H. Blomberg a écrit plusieurs poèmes sur le train :

Norrgående nattåget, Stora orons män, Stockholm : Bonnier, 1918, 114 p.

Natttåget, Jorden och jaget, Stokholm, 1924, 120 p.

De tva tågen, Jorden och jaget, Stokholm, 1924, $120 \mathrm{p}$.

Tredje klass, Jorden och jaget, Stokholm, 1924, $120 \mathrm{p}$.

19. Blomberg, H. : Nattåget, Jorden och jaget, Stokholm, 1924, 120 p.

20. Op. cit.

21. Sjögren, L. : Kalmar-Borås, Köttets hus, Stockholm : LT, 1971, 81 p.

22. Op. cit.

23. Op. cit.

24. La « saudade " portugaise est peut être la notion qui s'en rapproche le plus.

25. Op. cit.

26. Op. cit. 
27. Lindegren, E. : Arioso, Sviter, Stockholm : Bonnier, 1947, 167 p.

28. Op. cit.

29. Barthes, R. : Mythologies, Paris : Seuil, 1957, 267 p.

30. Ljungdal, A. : Fart, Ungdom, Stockholm : Bonnier, 1931, 78 p.

31. Fröding, G. : Tronskifte, Nya dikter, Stockholm : Bonnier, 1894, 117 p.

32. Hansson, O. : Tankar på nattsnälltåget, Efterlämnade skrifter, Hälsingborg, 1928, 191 p.

33. Op. cit.

\section{RÉSUMÉS}

Le propos de cet article est saisir la place du train dans la création poétique suédoise. L'intérêt poétique de ce moyen de transport prend ses racines au moment où les poètes du début du siècle montent dans le train et se réservent les places à la fenêtre. Ils s'abandonnent alors à la description de la nature scandinave et font du train le lieu où le paysage est roi. La fenêtre du compartiment offre au voyageur un panorama ambulant qui métamorphose le spectacle de la nature en laissant le voyageur découvrir des régions jusque là inconnues et en modifiant son regard. Et bien souvent, le train devient le lieu poétique de la rêverie par excellence. Le grondement inlassable des roues sur les rails rythme l'ennui confortable du voyage et incite à la rêverie nonchalante, une rêverie qui glisse immanquablement vers la nostalgie du temps révolu de l'enfance, thème profondément scandinave. Enfin, bouleversant les règles traditionnelles du temps et de l'espace, les poètes subissent les sensations de la vitesse maintenant accessible à tous. De cette expérience, naît une mythologie du train, machine à la fois magique et mystérieuse. Marquant ainsi le triomphe de l'homme sur la nature, le train des écrivains suédois, imprégnés de la philosophie de Kierkegaard, rapproche le poète de Dieu.

Dieser Beitrag hebt die Bedeutung der Eisenbahn in der schwedischen Dichtung hervor. Das Interesse für dieses Verkehrsmittel entsteht in dem Augenblick, als die Dichter um die Jahrhundertwende in den Zug steigen und einen Fensterplatz besetzen. Sie widmen sich dann der Beschreibung der schwedischen Natur und machen aus dem Abteil einen Ort, an dem die Landschaft im Vordergrund steht. Das Kupeefenster bietet dem Reisenden ein vorbeiziehendes Panorama dar, das die Ansicht der Natur verwandelt, es lässt ihn bis dahin unbekannte Gegenden entdecken und verändert den Blick. Oft wird der Zug zum Ort der poetischen Schöpfung und der Träumerei par excellence. Das unaufhörliche Rattern der Räder wird zur Geräuschkulisse der bequemen langweiligen Reise und fordert das müssige Dahinträumen, wobei die Träumerei unausweichlich in eine Sehnsucht zurück in die Zeit der verschwundenen Kindheit übergeht, und somit an ein sehr skandinavisches Motiv anknüpft. Ist nun einmal mit den herkömmlichen Begriffen von Zeit und Raum gebrochen worden, so nehmen die Dichter die jetzt von allen zu erlebende Geschwindigkeit wahr. Aus dieser Erfahrung erwächst eine Mythologie der Eisenbahn, einer magischen und geheimnisvollen Maschine. So signalisiert der Zug dem vom Kierkegaardschen Denken angeregten schwedischen Schriftsteller den Sieg des Menschen über die Natur, er bringt den Dichter näher zu Gott. 
AUTEUR

SYLVAIN BRIENS

Doctorant à l'Université de Paris IV-Sorbonne (département d'études scandinaves) 